\title{
Combining Declarative and Procedural Views in the Specification and Analysis of Product Families
}

\author{
Maurice H. ter Beek \\ ISTI-CNR, Pisa, Italy \\ LIACS, Leiden University, NL \\ maurice.terbeek@isti.cnr.it
}

\author{
Alberto Lluch Lafuente \\ IMT \\ Lucca, Italy \\ alberto.lluch@imtlucca.it
}

\author{
Marinella Petrocchi \\ IIT-CNR \\ Pisa, Italy \\ marinella.petrocchi@iit.cnr.it
}

\begin{abstract}
We introduce the feature-oriented language FLAN as a proof of concept for specifying both declarative aspects of product families, namely constraints on their features, and procedural aspects, namely design processes and run-time behaviour. FLAN is inspired by the concurrent constraint programming paradigm. A store of constraints allows one to specify in a declarative way all common constraints on features, including cross-tree constraints as known from feature models. A standard yet rich set of process-algebraic operators allows one to specify in a procedural way the configuration and behaviour of products. There is a close interaction between both views: (i) the execution of a process is constrained by its store to forbid undesired configurations; (ii) a process can query a store to resolve design and behavioural choices; (iii) a process can update the store, for instance to add new features. An implementation in the Maude framework allows for a variety of formal automated analyses of product families specified in FLAN, ranging from consistency checking to model checking.
\end{abstract}

\section{Categories and Subject Descriptors}

D.2.4 [Software Engineering]: Software/Program Verification-Formal methods, Model checking, Validation

\section{General Terms}

Design, Experimentation, Verification

\section{Keywords}

Product families, Variability, Process algebra, Concurrent constraint programming, Behavioural analyses, Maude

\section{INTRODUCTION}

Research on applying formal methods in SPLE traditionally focusses on modelling and analysing structural rather than behavioural constraints in product families. However,

Permission to make digital or hard copies of all or part of this work for personal or classroom use is granted without fee provided that copies are not made or distributed for profit or commercial advantage and that copies bear this notice and the full citation on the first page. To copy otherwise, to republish, to post on servers or to redistribute to lists, requires prior specific permission and/or a fee.

Copyright 2013 ACM ...\$15.00. many software-intensive systems are embedded, distributed and critical, making it important to be able to model and analyse also their behaviour, as a form of quality assurance. Recent years have witnessed a growing interest in specifically considering also the behavioural variability of product families. This has resulted in variants of UML diagrams 30, extensions of Petri nets 24, 25 and a variety of frameworks with transition system semantics $11,17,14,19,9,3$. As a result, behavioural analysis techniques such as model checking have become available for the verification of (temporal) logic properties of product families.

Specifying a product family directly in an operational model is often not easily feasible. Therefore it can be useful to resort to high-level formal languages with semantics over those operational models, as is common in the context of process algebra. Several extensions of CCS 23 have been proposed to model product families 12, 14, 15, 20, but none of these can combine behavioural constraints with all common structural constraints known from feature models.

We introduce here the feature-oriented language FLAN as a proof of concept for specifying product families by taking both structural and behavioural constraints into account. It is inspired by concurrent constraint programming 27. and its application in process algebra [7. A store of constraints allows one to specify in a declarative way all common structural constraints known from feature models, including cross-tree constraints. Moreover, a rich set of processalgebraic operators allows one to specify in a procedural way both the configuration and behaviour of products.

The declarative and procedural views are closely related: (i) the execution of a process is constrained by its store, e.g., to avoid introducing inconsistencies; (ii) a process can query a store in order to resolve options regarding the design and behaviour; (iii) a process can update the store, for instance to add new features.

Inspired by 12, we implemented FLAN in the executable modelling language Maude [10], whose rich toolkit enables the application of a variety of formal automated analysis techniques to product families specified in FLAN, from consistency checking to model checking.

The paper is organised as follows. Section 2 describes a running example of a family of coffee machines. In Sect. 3 we present the syntax and semantics of FLAN and a specification of the example. Section 4 illustrates the Maudesupported automated analyses of the example. We discuss related work in Sect. 5, report some concluding remarks in Sect. 6 and list promising future work in Sect. $7^{1}$

\footnotetext{
${ }^{1}$ For the convenience of the reviewers, an appendix contains
} 


\section{A FAMILY OF COFFEE MACHINES}

We use a popular running example in the style of $2,3,4$ 5, 9, 12, 24, 25. It describes a (simplified) family of coffee machines in terms of the following list of requirements:

1. Initially, a coin must be inserted: either a euro, exclusively for products for the European market, or a dollar, exclusively for Canadian products;

2. Upon the insertion of a coin, a choice for sugar must be offered, followed by a choice of beverages;

3. The choice of beverage (coffee, tea, cappuccino) varies, but every product must offer at least one beverage, tea may be offered only by European products, and all products that offer cappuccino must also offer coffee;

4. Optionally, a ringtone may be rung after the delivery of a beverage. However, a ringtone must be rung after serving a cappuccino;

5. After the beverage is taken, the machine returns idle.

These requirements define products by combining structural constraints defining valid feature configurations (e.g. "every product must offer at least one beverage") with temporal constraints defining valid behaviour, i.e. action sequences (e.g. "a ringtone must be rung after serving a cappuccino").

\section{FLAN: SYNTAX AND SEMANTICS}

The feature-oriented language FLAN we propose here is loosely inspired by the CCS-like process algebra CL4SPL presented in 12, but it strongly differs in its treatment of the cross-tree constraints known from feature models and in the separation of declarative and procedural aspects inspired by the concurrent constraint programming paradigm 27 and its adoption in process calculi [7].

The core notions of FLAN are those of features, constraints, processes and fragments, each of which can be identified in the syntax of FLAN presented in Fig. 1 More precisely, features range over $f$ and $g$ and constraints, processes and fragments correspond to the syntactic categories $S, P$ and $F$, respectively.

Features. A feature is a term describing specific elements or properties of a product. The universe of features is denoted by $\mathcal{F}$. The features of our running example are the coins accepted (i.e. euro and dollar), the products offered (i.e. coffee, tea and cappuccino) and additional elements such as sugar (the capability to regulate the quantity of sugar in a product) and ringtone (the capability to emit an audio signal).

Constraints. The declarative part of FLAN is represented by a store of constraints that defines both constraints on features extracted from the product requirements and additional information (e.g. information about the context where the product will operate).

Two important notions of constraint stores are consistency (which amounts to logical satisfiability of all constraints forming a store in our case) and entailment $S \vdash c$ of a constraint $c$ in a store $S$ (which amounts to logical entailment in our case).

the complete Maude implementation of our case study.

$$
\begin{aligned}
F & ::=[S|| P] \\
S, T & ::=K|f \triangleright g| f \otimes g|S T| \top \mid \perp \\
P, Q & ::=0|X| A . P|P+Q| P ; Q|P| Q \\
A & ::=\text { install }(f)|\operatorname{ask}(K)| a \\
K & ::=p|\neg K| K \vee K
\end{aligned}
$$

where $a \in \mathcal{A}, p \in \mathcal{P}$ and $f, g \in \mathcal{F}$

Figure 1: The syntax of FLan

A constraint store is any term generated by $S$ in the grammar of FLAN. The most basic constraint stores are $T$ (no constraint at all), $\perp$ (inconsistent) and ordinary boolean propositions (generated by $K$ ). Constraints can be combined by juxtaposition (whose semantics amounts to logical conjunction).

We assume that the standard structural constraints on features (such as options, obligations and alternatives) are expressed using boolean propositions (e.g. as explained in 28] ) For this purpose, we assume that the universe $\mathcal{P}$ of propositions contains a Boolean predicate has $(\cdot): \mathcal{F} \rightarrow \mathbb{B}$ that can be used (in grounded form) to denote the presence of a feature in a product. Boolean propositions can also be used to represent additional information such as contextual facts. Examples from our running example are in (Europe) and in (Canada), respectively used to state the fact that the coffee machine being configured is meant to be used in Europe or in Canada. Boolean propositions can state relations between contextual information and features, like in (Europe) $\rightarrow$ has (euro) (i.e. a coffee machine for the European market needs a euro coin slot).

Cross-tree constraints, instead, are handled as first-class citizens. A constraint $f \triangleright g$ expresses that feature $f$ requires the presence of feature $g$ while a constraint $f \otimes g$ expresses that features $f$ and $g$ mutually exclude each other's presence (i.e. they are incompatible). Of course, also these constraints can be encoded as boolean propositions. For instance, $f \otimes g$ and $f \triangleright g$ can be equivalently expressed as has $(f) \leftrightarrow \neg$ has $(g)$ and has $(f) \rightarrow$ has $(g)$, respectively. We can use indeed such logical encoding to reduce consistency checking and entailment to logical satisfiability (and hence exploit Maude's SAT solver). However, their first-class treatment allows us to emphasize some important choices in the semantics of the language (cf. the discussion of rule INST later on).

We also consider a class of action constraints, reminiscent of Featured Transitions Systems 9], where transitions are subject to the presence of features. For instance, in a coffee machine equipped with a slot for euro coins we will use euro for the action of inserting a euro coin and do(euro) as a proposition stating the execution of that action. The relations between the action euro and the presence of the corresponding feature euro can be formalised as $d o($ euro $) \rightarrow$ has (euro), i.e. the insertion of a euro coin requires the presence of an appropriate coin slot. In general, we assume that each action $a$ may have a constraint $d o(a) \rightarrow p$. Such constraints act as a sort of guard to allow or forbid the execution of actions (as illustrated later on in the discussion of rule ACT).

The constraint store $S$ in Fig. 4 formalises part of the requirements specified in Sect. 2 for our running example. It contains both contextual information (e.g. in(Europe)) 


$$
\begin{array}{rlllll}
P+Q & \equiv Q+P & P+(Q+R) & \equiv & (P+Q)+R \\
P \mid Q & \equiv Q \mid P & P \mid(Q \mid R) & \equiv & (P \mid Q) \mid R \\
P+0 & \equiv P & P ;(Q ; R) & \equiv(P ; Q) ; R \\
0 ; P & \equiv P & P ; 0 & \equiv P \\
P \mid 0 & \equiv P & P & \equiv P\left[{ }^{Q} / X\right] \text { if } X \doteq Q
\end{array}
$$

Figure 2: Structural congruence in FLan

and action constraints (e.g. do(euro) $\rightarrow$ has (euro)). Note, for instance, that from requirement 1 we understand that euro and dollar are mutually exclusive features (formalized as dollar $\otimes$ euro), while from requirement 3 we understand that cappuccino requires coffee (formalized as cappuccino $\triangleright$ coffee).

Processes. The procedural part of FLAN is represented by processes. A process can be one of the following:

- 0 , the empty process that can do nothing;

- $X$, where $X$ is a process identifier. We assume that there is a set of process definitions of the form $X \doteq P$. We also assume that recursively defined processes are finitely branching, which can be ensured in standard ways (e.g. prefixing every occurrence of a process identifier or every process definition with an action);

- A.P, a process willing to perform the action $A$ and then to behave as $P$;

- $P+Q$, a process that can non-deterministically choose to behave as $P$ or as $Q$;

- $P ; Q$, a process that must progress first as $P$ and then as $Q$;

- $P \mid Q$, a process formed by the parallel composition of $P$ and $Q$, which evolve independently.

It is worth remarking that we distinguish between ordinary actions (from a universe $\mathcal{A}$ ) and the special actions install $(f)$ (used to denote the installation of a feature $f$ ) and ask $(K)$ (used to query the store). We shall see that each such kind of action is treated differently in rules of the operational semantics.

In our example, we will consider the following actions: euro and dollar (insertion of the respective coin); sugar (sugar selection); coffee, tea, and cappuccino (beverage selection); and ringtone (ringtone emission).

Fragments. Finally, a fragment $F$ is a term $[S \| P]$, composed by a store of constraints $S$ and a process $P$. Each of the components of a fragment may influence each other, along the lines of the concurrent constraint programming paradigm 27]: a process may update its store which, in turn, may condition the execution of process actions.

The operational semantics of of closed fragments (i.e. its reduction semantics) is formalised in terms of the state transition relation $\rightarrow \subseteq \mathbb{F} \times \mathbb{F}$ illustrated in Figure 3, where $\mathbb{F}$ denotes the set of all terms generated by $F$ in the grammar of Figure 1] Technically, such reduction relation is defined in Structural Operational Semantics (SOS) style (i.e. by induction on the structure of the terms denoting a fragment)
(Inst) $\frac{\forall g \in \mathcal{F} . S \vdash f \otimes g \Rightarrow S \not \forall \text { has }(g)}{[S \| \text { install }(f) . P] \longrightarrow[S \text { has }(f) \| P]}$

(ASK) $\frac{S \vdash K}{[S \| \operatorname{ask}(K) \cdot P] \longrightarrow[S \| P]}$

$(\mathrm{ACT}) \frac{S \vdash(d o(a) \rightarrow K) \Rightarrow S \vdash K}{[S \| a . P] \longrightarrow[S \| P]}$

$(\mathrm{OR}) \frac{[S \| P] \longrightarrow\left[S^{\prime} \| P^{\prime}\right]}{[S \| P+Q] \longrightarrow\left[S^{\prime} \| P^{\prime}\right]}$

$(\mathrm{SEQ}) \frac{[S \| P] \longrightarrow\left[S^{\prime} \| P^{\prime}\right]}{[S \| P ; Q] \longrightarrow\left[S^{\prime} \| P^{\prime} ; Q\right]}$

$(\mathrm{PAR}) \frac{[S \| P] \longrightarrow\left[S^{\prime} \| P^{\prime}\right]}{[S \| P \mid Q] \longrightarrow\left[S^{\prime} \| P^{\prime} \mid Q\right]}$

Figure 3: Reduction semantics of FLan

modulo a structural congruence relation $\equiv \subseteq \mathbb{F} \times \mathbb{F}$. As usual such reduction relation implicitly defines an unlabeled transition system.

Considering terms up to a structural congruence allows us to identify the different ways of denoting the same fragment. In our case we consider the least congruence on fragments closed with respect to the commutativity and associativity of non-deterministic and parallel composition of processes; the associativity of sequential composition of processes; the identity of the non-deterministic choice, sequential and parallel composition of processes; and the expansion of recursive process definitions. The choice of the axioms (some of which may seem unsual) is not an accident. Indeed, they all can be naturally and efficiently treated by Maude so that our semantics enjoys several good properties: (1) it is (efficiently) executable; (2) each semantic rule of Figure 3 corresponds exactly to one conditional rewrite rule in the Maude implementation of FLAN; and (3) the number of reduction rules is small and thus the semantics and its implementation are compact and easy to read.

As usual, reduction rules are expressed in terms of a set of (possibly empty) premises (above the line) and a conclusion (below the line).

Rules INST and ACT are very similar, both allowing a process to execute an action if certain local constraints are satisfied. This notion of local consistency allows us to let processes progress even if their stores are temporarily inconsistent. This means that, contrary to classical concurrent constraint programming, we allow processes to run with inconsistent stores. This is the main reason why we do not use a general tell-like operation as in 27, 7]. In particular, rule INST forbids inconsistencies with respect to exclude constraints, and rule ACT forbids inconsistencies with respect to action constraints. Other inconsistencies, e.g. due to require constraints or basic constraints, are temporarily allowed and cannot block a process. This allows us to deal with stores containing constraints such as mutually requiring features (e.g. $f \triangleright g$ and $g \triangleright f$ ) and to describe processes that may install them in any order. The eventuality of consistency can be verified by resorting to reachability or model checking analysis as we shall see in the next section.

Rule Ask formalises the semantics of the usual ask $(\cdot)$ 


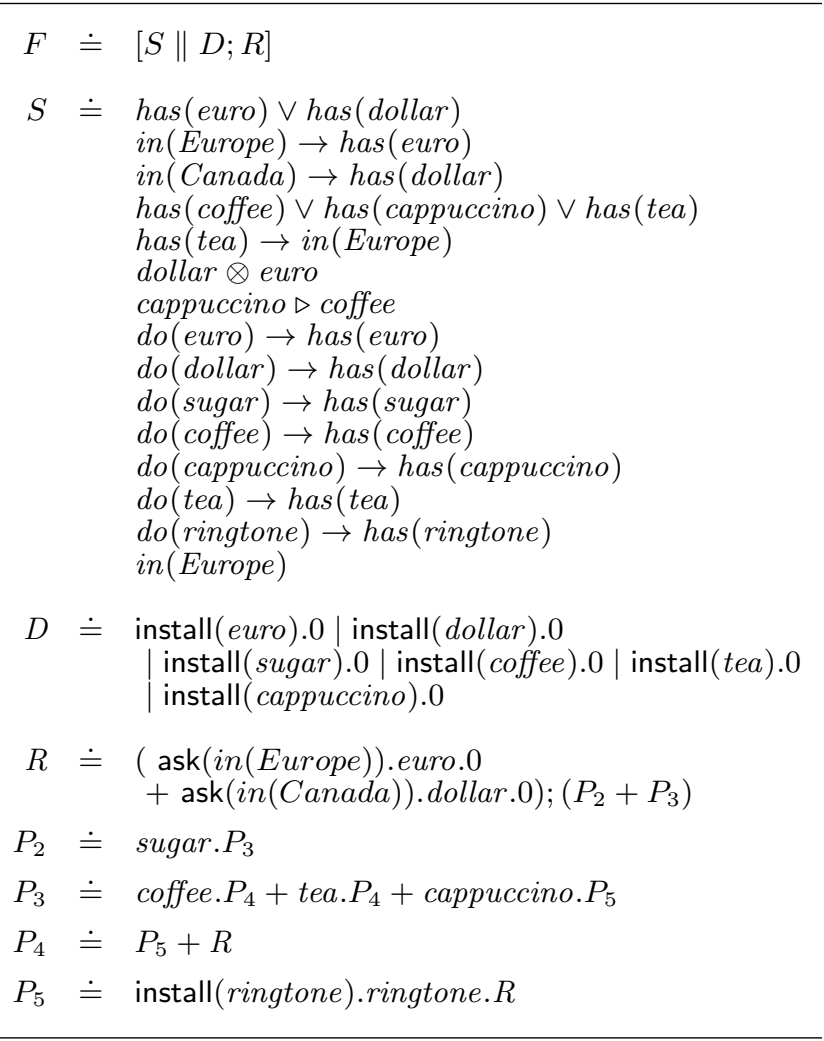

Figure 4: Initial specification of the coffee machine

operation as known from concurrent constraint programming 27. It allows to block a process until a proposition can be derived from the store.

Rule OR is quite straightforward. It allows the process to evolve as any of the branches. It is worth remarking that non-determinism can be solved at the procedural level (by relying on ask $(\cdot)$ actions) or at the declarative level (by using a non-deterministic choice that may be solved by the constraint store), thus providing a great flexibility to fragment designers (as illustrated later on).

Rules SEQ and PAR are standard. The former formalises the usual sequential composition, while the latter formalises an interleaving parallelism.

Example. Figure 4 shows an initial comprehensive specification of the coffee machine. The fragment $F$ is composed by the store $S$ and the concatenation of two processes, namely $D$, which specifies an initial design phase, and $R$, which specifies the run-time behaviour of the coffee machine.

The design process $D$ is quite simple. It is just formed by the parallel composition of the installation of all the features that the coffee machine may exhibit. This specifies a sort of race between features and may be thought of as independent designers competing to install the features they are responsible for. Of course, not all executions may end up with a consistent configuration (as we will see in the next section). Indeed, while the semantics of FLAN forbids inconsistencies due to exclude constraints, all other potential sources of inconsistencies are not forbidden.

Process $R$ describes the run-time operation of the coffee

$$
\begin{aligned}
F \doteq & {\left[S \mid D^{\prime} ; R^{\prime}\right] } \\
D^{\prime} \doteq \quad & \quad(\text { install }(\text { euro }) .0+\text { install }(\text { dollar }) .0) \\
& \quad \mid \text { install }(\text { sugar }) .0 \mid \text { install }(\text { coffee }) .0 \mid \text { install }(\text { tea }) .0 \\
& \mid \text { install }(\text { cappuccino }) .0
\end{aligned}
$$

Figure 5: Final specification of the coffee machine

machine. Depending on the country it is meant for, the machine may either accept a euro or a dollar. After that, it may be subject to a sugar regulation $\left(P_{2}\right)$ or not $\left(P_{3}\right)$. The next step is the beverage selection and delivery, which may be followed by a ringtone $\left(P_{5}\right)$ or not, after which it returns to its initial state.

It is worth to note that $D$ and $R$ are not pure design and run-time processes: indeed feature ringtone is not installed by $P$ but by $P$. In other words, the feature ringtone is dynamically installed and it can be thought, for instance, as a software module. This is an interesting example of a partial design process where some non-mandatory features are not installed and products are only partially configured, and a run-time configurable process that installs features when needed.

In the next section, we will see that this specification has some flaws that can be spotted with our implementation in Maude. This will eventually lead to the corrected specification that follows from the modified parts depicted in Fig. 5.

\section{MAUDE: AUTOMATED ANALYSES}

In this section we describe some automated analysis activities supported by the implementation of our approach in Maude's formal environment.

We illustrate the use of some of the tools in what could be a typical specification and analysis life-cycle of a product family within our framework: (i) an initial constraint store (capturing the feature constraints described in the requirements) is specified and checked for consistency; (ii) a design process is specified and executed step-by-step; (iii) a consistency check is performed on all possible configurations allowed by the design process; and (iv) the product behaviour is specified and checked with respect to its requirements (that may include temporal requirements in addition to features constraints). We underline that this is only an example. The tools and techniques we illustrate can be combined and applied in many other ways.

Checking the consistency of the initial constraints. The consistency of a store is implemented by a function consistent that, given a constraint store, returns true if the store is consistent and false otherwise. This function can be used to check, e.g., the consistency of the initial store S presented in Fig. 4 as follows.

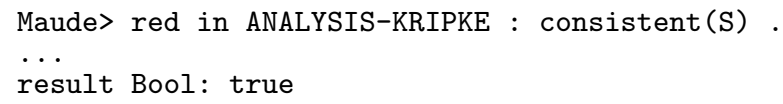

The result confirms that the initial store $\mathbf{S}$ is consistent. 
Executing the design process. Starting from a consistent or inconsistent store, the user may want to specify and execute a design process that ends up with a maximally configured product. Consider for instance the initial store $\mathbf{S}$ and the design process D presented in Fig. 4

The Maude command rew can be used to execute the fragment $[\mathrm{S} \mid \mathrm{D}]$ as follows.

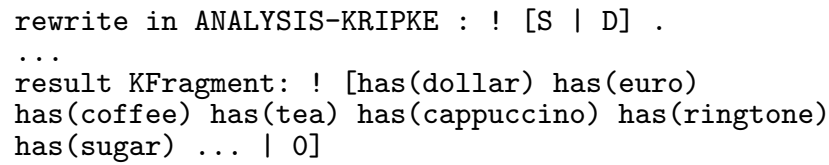

The fragment runs until the underlying process becomes the empty process resulting in a product configured with several features (for ease of readiness the part of the store that has not changed is abbreviated with ....). Clearly, such configuration is inconsistent since it contains mutually exclusive features (euro and dollar coin slots). We will see how to automatically spot such inconsistencies.

Checking the consistency of all configurations. Indeed, an interesting analysis at this point is concerned with the consistency of all possible obtained products.

We recall that the design process may pass through several intermediate states where the store of constraints is inconsistent. This can be checked using the reachability command reach as follows:

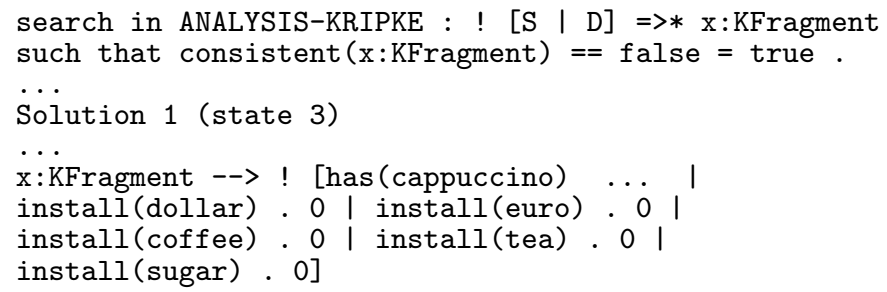

The first state we get, for instance, is one where the feature cappuccino has been installed. It is inconsistent because of the requirement to have coffee as well, which is yet to be installed. Clearly, such temporary inconsistencies are acceptable but one would expect all possible final products to be consistent. We can use again the search command for this purpose, looking for a reachable final and inconsistent state as follows.

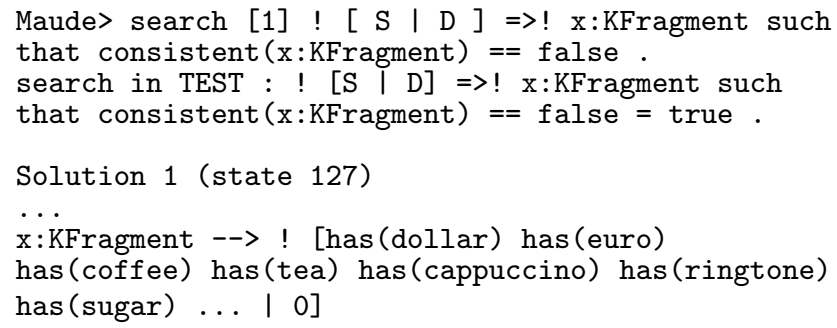

The analysis provides the final yet inconsistent store we obtained before by executing the design process. We can now obtain a witness of the inconsistency with the function inconsistency.

Maude> red in ANALYSIS-KRIPKE : inconsistency has (dollar) has (euro) has (coffee) has(tea) has (cappuccino) has (sugar) ... ).

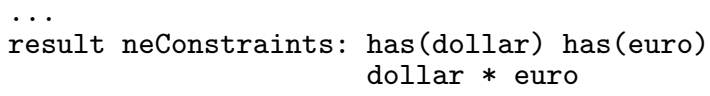

The analysis spots the above mentioned inconsistency of installing two mutually excluding features (the euro and dollar coin slots) by reporting the subset of constraints formed by has(dollar), has(euro) and dollar $\otimes$ euro.

We can fix this issue and produce a new design process $D^{\prime}$ (cf. Fig. 5) in which the installation of euro and dollar coin slots is controlled procedurally through a non-deterministic choice. We can verify as follows that this new process produces consistent stores only.

Maude> search [1] in ANALYSIS-KRIPKE : ! [ S | D' ] $=>! \mathrm{x}:$ KFragment such that consistent $(\mathrm{x}: \mathrm{KFragment})$ $==$ false. search in ANALYSIS-KRIPKE : ! [S | D'] $\Rightarrow$ ! $\mathrm{x}:$ KFragment such that consistent $(\mathrm{x}:$ KFragment $)==$ false $=$ true .

No solution.

states: 96

Checking behavioural properties. After fixing the specification of the design we can analyse the run-time behaviour of the product. We can now check, for instance, that the runtime behaviour does not introduce inconsistencies by using the LTL model checker of Maude. The property we check is $\langle>$ [] isConsistent, i.e. eventually consistency becomes an invariant.

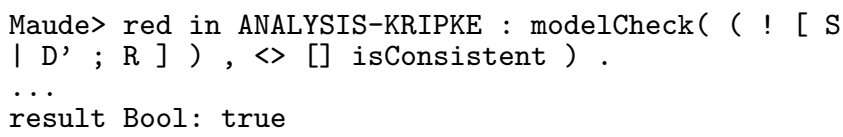

The results confirms that consistency is eventually guaranteed and preserved during the run-time operation of the coffee machine.

We may however notice that the conditional statement used to accept a dollar or a euro is actually redundant due to the introduced constraints. A possible, simpler run-time process is $R^{\prime}$ (cf. Fig. 5). It is very much like $R$, but the conditional statement has been replaced by a non-deterministic choice that will be consistently solved at run-time due to the presence of the action constraints do(euro $) \rightarrow$ has $($ euro $)$ and do(dollar $) \rightarrow$ has (dollar $)$ in the store, which will forbid the use the actions euro or dollar if the corresponding feature has not been installed. This time, contrary to what we did earlier for the design process, we are replacing procedural information by declarative information. The resulting process enjoys the property of eventually preserving consistency, which can be checked as follows.

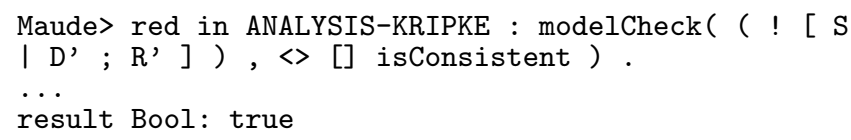

The results confirms that consistency is still preserved during the run-time operation of the coffee machine.

The LTL model checker can of course be used to check additional requirements. For instance, we can check that the temporal requirement 4 of our case study (i.e. " $a$ ringtone must be rung after serving a cappuccino" ringtone is activated after serving a cappuccino as follows. 
Maude> red in ANALYSIS-LTS : modelCheck ( ( ! (\{do('machine) $\}[S \mid D ' ; R '])),[]$ (\{cappuccino $\}$ $\rightarrow<>$ ringtone $\}$ ) ) .

...

result Bool: true

The results confirms that a ringtone eventually follows (the delivery of) a cappuccino.

\section{RELATED WORK}

There is an increasing body of research on how to successfully apply automated behavioural verification techniques, like model checking, in the particular context of (software) product families. The challenge, to the best of our knowledge first recognized in 21, 22, is to develop formal and modular modelling and verification approaches which specifically take cross-cutting feature constraints into account. In this section, we discuss a number of formal methods and analysis techniques that have been applied in SPLE.

There are two well-known lines of research on modelling product families in terms of extensions of LTSs, which both define family behaviour as actions (features) and use advanced model-checking techniques for the verification of behavioural properties. One makes use of extensions of Modal Transition Systems (MTSs) 11, 17, 19, 3, the other of Featured Transition Systems (FTSs) 9 .

Modal Transition Systems. MTSs 18 were recognised as a suitable behavioural model for describing product families in 11. A fixed-point algorithm, implemented in a tool, is defined to check whether an LTS conforms to an MTS with respect to several different branching relations. In the context of SPLE, it allows to check the conformance of the behaviour of a product against that of its product family.

VMC (http://fmt.isti.cnr.it/vmc/) 4, 5, is a tool for modelling and analysing behavioural variability in product families modelled as MTSs 3]. VMC thus accepts a product family specified as an MTS, possibly with additional variability constraints, after which it allows the user to interactively explore this MTS; efficiently model check properties (branching-time temporal logic formulae) over an MTS; visualise the (interactive) explanations of a verification result; automatically generate one, some, or all of the family's valid products (represented as LTSs); browse and explore these; efficiently model check whether or not products (one, some, or all) satisfy certain properties; and, finally, help the user to understand why a certain valid product does or does not satisfy specific verified properties, by allowing such a product to be inspected individually.

Feature Transition Systems. An FTS 9] is a doubly labelled transition system with an associated feature diagram. Its states are labelled with atomic propositions, while a specific distinction among its transitions is obtained through an edge-labelling indicating which transitions correspond to which features.

SNIP 8] is a model checker for product families modelled as FTSs specified in a language based on that of the SPIN model checker (http://spinroot.com/). Features are declared in the Text-based Variability Language (TVL) and are taken into account by the explicit-state model-checking algorithm of SPIN for verifying properties expressed in fLTL (feature LTL) interpreted over FTSs (e.g. to verify a property over only a subset of the set of all valid products). Ex- haustive model-checking algorithms (which continue their search also after a violation was found) moreover allow to verify all products of a family at once and to output all of the products that violate a property. Unlike VMC, SNIP is a command-line tool without a GUI. SNIP, however, treats features as first-class citizens, with built-in support for feature diagrams, and it implements model-checking algorithms specifically tailored for product families.

The tool suite SPLVERIFIER [1] uses standard off-the-shelf model-checking techniques to verify the absence of feature interactions by means of an approach called feature-aware verification. To this aim, the AutoFeAture automata language for specifying features in separate and composable units was developed, while a variant of abstract syntax trees, called Feature Structure Trees (FSTs), forms the basis for encoding the variability. SPLVERIFIER offers two methods: a brute-force one generates and verifies all valid products, while an alternative one avoids the generation of all individual products as it verifies all possible feature combinations on a single product that is purpose-built to contain all the family's features. Like SNIP, features are central to SPLVERIFIER, but only the (renowned) problem of detecting feature interactions is addressed. Unlike VMC and SNIP, behavioural variability is not considered.

In this paper, we proposed to specify product families in a high-level formal process-algebraic language, FLAN, which has transition systems as semantic domain. While, in principle, product family behaviour could be directly specified using transition systems from a practical point of view it is more convenient to resort to some more intuitive linguistic formalism. In fact, when used as a specification formalism, transition systems are too low level and, above all, suffer from the lack of compositionality - in the sense that they offer no means for constructing the transition system of a (sub)family in terms of that of its components. On the contrary, the process-algebraic linguistic terms offered by FLAN are more intuitive and concise notations. Using them, product families can be built in a compositional way. Like the approach based on FTSs, we thus use a high-level language for modelling, treating features as first-class citizens, and a transition system semantics for analysis. While we currently use Maude for the automated verification of behavioural properties of product families specified in FLAN, in the future we hope to make their semantic models (LTSs, basically) amenable to model checking with VMC. FLAN is loosely inspired by the CCS-like process algebra CL4SPL presented in 12. Unlike FLAN, however, CL4SPL has no language constructs for the cross-tree constraints known from feature models nor a store of constraints to separate the declarative aspects of a product family from its procedural aspects.

Process algebras and Petri nets. A process-algebraic theory for the modelling and analysis of product families was developed also in $14,15,20$. PL-CCS extends CCS by a variant operator that allows to model alternative behaviour in the form of alternative processes, with the meaning that only one of the alternative processes will exist at run-time. PLCCS has a SOS semantics defined over multi-valued MTSs. To reason on the behaviour of product families specified in PL-CCS, a multi-valued version of the modal $\mu$-calculus is defined, i.e. the interpretation of a logic formula over a product family no longer yields true or false, but rather a set of 
configurations characterising exactly those products of the family which satisfy the behavioural property under verification. Unlike FLAN, PL-CCS however does not cater for the cross-tree constraints known from feature models. Also, the analysis is limited to verification by model checking which is moreover not implemented.

The same idea underlying FTSs, namely to explicitly label the transitions of an LTS with the set of features (i.e. products) for which the transition is available, was also applied to Petri nets in 24, 25, resulting in feature (Petri) nets. Larger feature nets can be constructed from smaller ones to model the addition of new features to a product family, while correctness criteria can ensure that the resulting composition preserves the original behaviour. An extension can capture the dynamic reconfiguration of products by associating to each transition of a feature net also an update expression that describes how the feature selection evolves after firing (executing) the transition. The resulting feature reconfiguration model may remain disconnected from the ordinary behavioural model, thus offering orthogonality but at the same time allowing the reconfiguration to depend upon the underlying behaviour and vice versa. This has some similarities with the combination of declarative and procedural views that is at the heart of FLAN. Efficient formal analysis and verification techniques from Petri nets of course become available to feature nets, but their application in the specific context of product families has not yet been studied.

In 29, FTSs are translated into so-called adaptable featured Petri nets, after which projection and reachability techniques from Petri nets become available for product derivation and liveness analysis.

Other works. In 16, FTSs (including their associated feature diagrams) are translated into Maude specifications by graph transformation. Starting from a set of requirements, this means that first a feature diagram needs to be extracted (to model the variability) and only then the desired runtime behaviour can be specified (as an FTS). FLAN, on the contrary, allows to combine the specification of design and run-time processes directly from a given set of requirements, which may be very convenient, for instance to specify the behaviour of partially configured or run-time configurable products. Another difference is that the semantic foundation of our approach is based on techniques from concurrent constraint programming and process algebras rather than graph transformation. In [13, a feature-oriented approach to modelling product families in Event-B by means of a chain of refinements is explored by applying existing EventB (de)composition techniques to two case studies, using a prototypical feature composition tool. Behavioural variability is not considered, but it would be interesting to explore the feasibility of using this Feature Event-B as a high-level specification language on top of one of the aforementioned semantic models.

\section{CONCLUDING REMARKS}

We have introduced the feature-oriented language FLAN as a proof of concept for specifying and analysing both declarative and procedural aspects of product families.

We do not envisage FLAN to become the feature-oriented language, but we advocate that some of its features are very convenient and may be adopted by existing languages.

First, we think that the concurrent constraint program- ming paradigm provides a flexible mechanism for separating and (when necessary) combining declarative and procedural aspects. For instance, design decisions can be delayed to run-time, which is very convenient for software product families where features may be added while the system operates. Furthermore, the run-time specification can be discharged from design decisions such as feature constraints thus resulting in light-weight, understandable specifications.

Second, the implementation of FLAN in Maude allows one to exploit the rich analysis toolset of this framework. In this paper, we have essentially restricted ourselves to its SAT solver, its reachability analyser and its LTL model checker. However, there are other Maude tools whose use may be worth investigating. The statistical model checker PVESTA, for instance, could be used for evaluating the performance of product families in variants of FLAN with stochastic and quantitative aspects.

\section{FUTURE WORK}

We envisage several potentially interesting extensions of FLAN. For one, we can adopt further primitives and mechanisms from the concurrent constraint programming tradition. The concurrent constraint $\pi$-calculus [7], for instance, provides synchronisation mechanisms typical of mobile calculi (i.e. name passing), a check operation to prevent inconsistencies, a retract operation to remove (syntactically present) constraints from the store and a general framework for soft constraints (i.e. not only boolean). Such features have been shown successful for the specification of service level agreements and negotiation processes [6]. This may thus turn out to be useful when product families are to be designed by cooperating partners and are hence subject to negotiation mechanisms.

Another promising line of research is to provide an FTS and an MTS semantics of FLAN so that (i) FLAN becomes a high-level language for those semantic models and (ii) we can exploit the specialised analysis tools developed for them.

\section{Acknowledgements}

Maurice ter Beek conducted his work while on sabbatical leave at Leiden University. He gratefully acknowledges the hospitality and support during his stay in Leiden.

Maurice ter Beek and Alberto Lluch Lafuente were supported by the Italian Ministry of Instruction, University and Research project CINA (PRIN 2010LHT4KM), and by the European project QUANTICOL (FP7 600708).

Alberto Lluch Lafuente is also supported by the European projects ASCEns (FP7 257414) .

Marinella Petrocchi acknowledges the European projects NESSoS (FP7 256980) and Aniketos (FP7 257930).

\section{REFERENCES}

[1] S. Apel, H. Speidel, P. Wendler, A. von Rhein, and D. Beyer. Detection of feature interactions using feature-aware verification. In ASE'11. IEEE, 2011, 372-375.

[2] P. Asirelli, M. H. ter Beek, A. Fantechi, and S. Gnesi. A Logical Framework to Deal with Variability. In IFM'10, LNCS 6396. Springer, 2010, 43-58.

[3] P. Asirelli, M. H. ter Beek, A. Fantechi, and S. Gnesi. Formal Description of Variability in Product Families. In SPLC'11. IEEE, 2011, 130-139. 
[4] M. H. ter Beek, S. Gnesi, and F. Mazzanti. Demonstration of a model checker for the analysis of product variability. In SPLC'12. ACM, 2012, 242-245.

[5] M. H. ter Beek, F. Mazzanti, and A. Sulova. VMC: A Tool for Product Variability Analysis. In FM'12, LNCS 7436. Springer, 2012, 450-454.

[6] M. G. Buscemi and U. Montanari. QoS negotiation in service composition. Journal of Logic and Algebraic Programming 80, 1 (2011), 13-24.

[7] M. G. Buscemi and U. Montanari. CC-Pi: A Constraint-Based Language for Specifying Service Level Agreements. In ESOP'07, LNCS 4421. Springer, 2007, 18-32.

[8] A. Classen, M. Cordy, P. Heymans, A. Legay, and P.-Y. Schobbens. Model Checking Software Product Lines with SNIP. Software Tools for Technology Transfer 14, 5 (2012), 589-612.

[9] A. Classen, P. Heymans, P.-Y. Schobbens, A. Legay, and J.-F. Raskin. Model Checking Lots of Systems. In ICSE'10. ACM, 2010, 335-344.

[10] M. Clavel et al., Eds. All About Maude, LNCS 4350. Springer, 2007.

[11] D. Fischbein, S. Uchitel, and V. A. Braberman. A Foundation for Behavioural Conformance in Software Product Line Architectures. In ROSATEA'06. ACM, 2006, 39-48.

[12] S. Gnesi and M. Petrocchi. Towards an Executable Algebra for Product Lines. In FMSPLE'12. ACM, 2012, 66-73.

[13] A. Gondal, M. Poppleton, and M. Butler. Composing Event-B Specifications - Case-Study Experience. In $S C$ '11, LNCS 6708. Springer, 2011, 100-115.

[14] A. Gruler, M. Leucker, and K. D. Scheidemann. Modeling and Model Checking Software Product Lines. In FMOODS'08, LNCS 5051. Springer, 2008, 113-131.

[15] A. Gruler, M. Leucker, and K. D. Scheidemann. Calculating and Modeling Common Parts of Software Product Lines. In SPLC'08. ACM, 2008, 203-212.

[16] K. Khalfaoui, A. Chaoui, C. Foudil, and E. Kerkouche. Formal Specification of Software Product Lines: A Graph Transformation Based Approach. Journal of Software 7, 11 (2012), 2518-2532.
[17] K. G. Larsen, U. Nyman, and A. Wąsowski. Modal I/O Automata for Interface and Product Line Theories. In ESOP'O7, LNCS 4421. Springer, 2007, 64-79.

[18] K. G. Larsen and B. Thomsen. A modal process logic. In LICS'88. IEEE, 1988, 203-210.

[19] K. Lauenroth, K. Pohl, and S. Töhning. Model Checking of Domain Artifacts in Product Line Engineering. In ASE'09. IEEE, 2009, 269-280.

[20] M. Leucker and D. Thoma. A Formal Approach to Software Product Families. In ISoLA'12, LNCS 7609. Springer, 2012, 131-145.

[21] H. C. Li, S. Krishnamurthi, and K. Fisler. Verifying Cross-Cutting Features as Open Systems. In FSE'02. ACM, 2002, 89-98.

[22] H. C. Li, S. Krishnamurthi, and K. Fisler. Modular Verification of Open Features Using Three-Valued Model Checking. Automated Software Engineering 12 (2005), 349-382.

[23] R. Milner. Communication and Concurrency. Prentice Hall, 1989.

[24] R. Muschevici, D. Clarke, and J. Proença. Feature Petri Nets. In FMSPLE'10. Univ. of Lancaster, 2010.

[25] R. Muschevici, J. Proença, and D. Clarke. Modular Modelling of Software Product Lines with Feature Nets In SEFM'11, LNCS 7041. Springer, 2011, 318-333.

[26] G. D. Plotkin. A structural approach to operational semantics. Journal of Logic and Algebraic Programming 60-61 (2004), 17-139.

[27] V. A. Saraswat and M. C. Rinard. Concurrent Constraint Programming. In POPL'90. ACM, 1990, 232-245.

[28] P.-Y. Schobbens, P. Heymans, and J.-C. Trigaux. Feature Diagrams: A Survey and a Formal Semantics. In RE'06. IEEE, 2006, 136-145.

[29] H. Zhang, H. Zou, F. Yang, and R. Lin. Modeling and Analysis of Behavioral Variability in Product Lines. Journal of Information ES Computational Science 9, 12 (2012), 3589-3600.

[30] T. Ziadi and J. M. Jézéquel. Software Product Line Engineering with the UML: Deriving Products. In SPLC'06. Springer, 2006, 557-588. 


\section{APPENDIX}

\section{Implementation}

This section documents the complete specification of our implementation of FLAN in Maude.

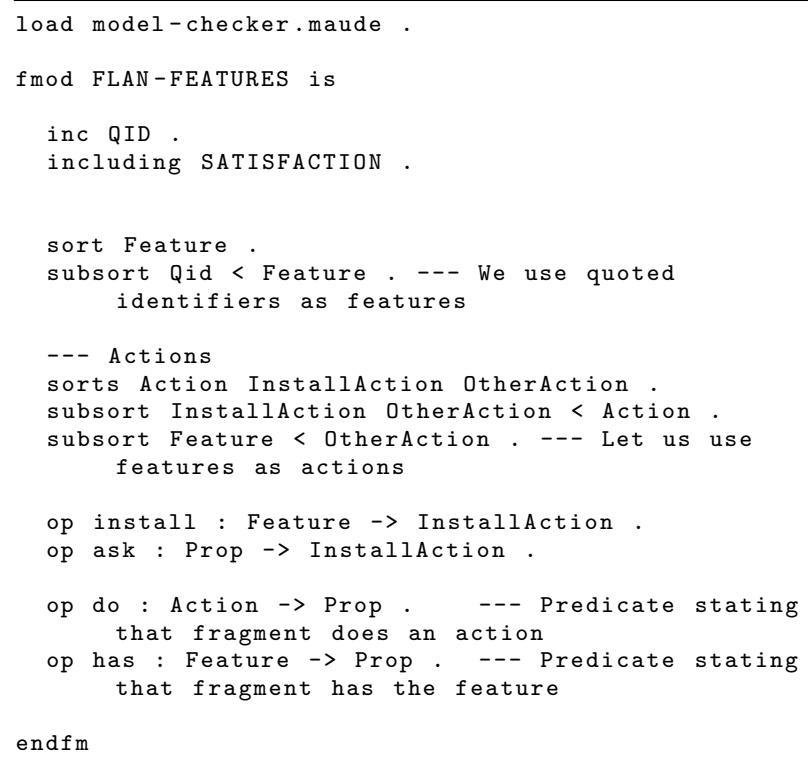

\section{fmod FLAN-CONSTRAINTS is}

pr FLAN-FEATURES.

pr SAT-SOLVER

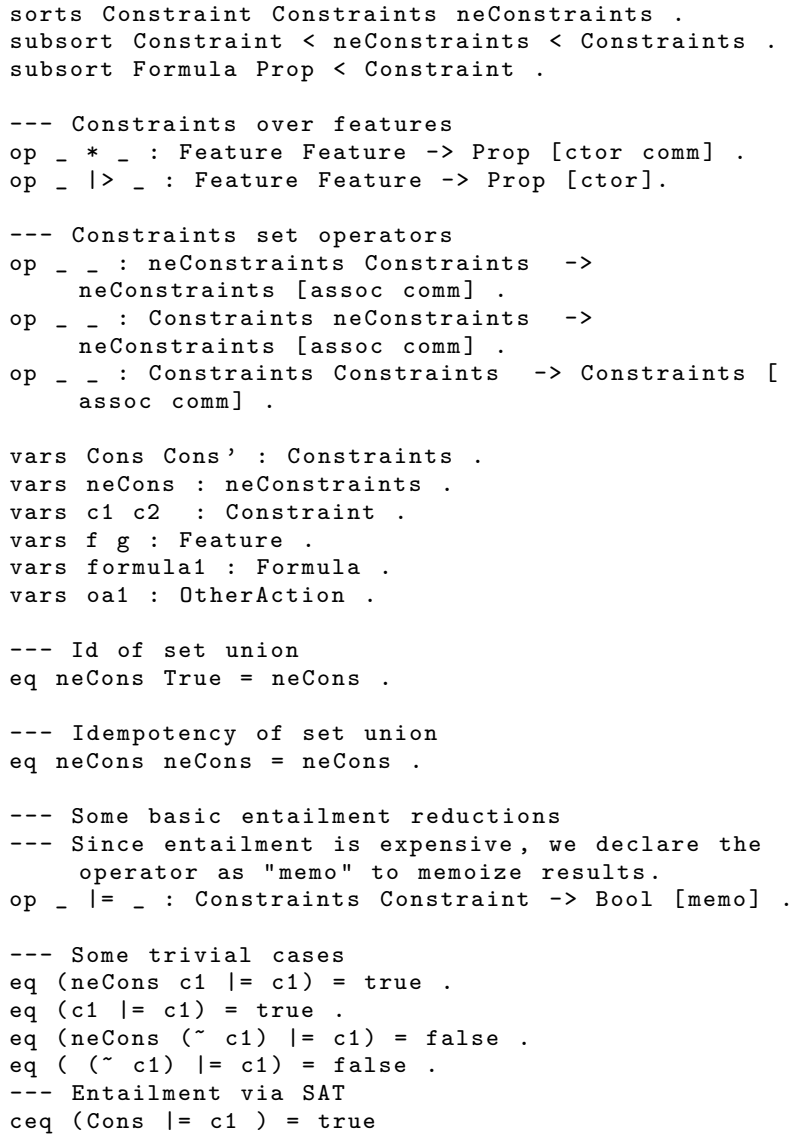

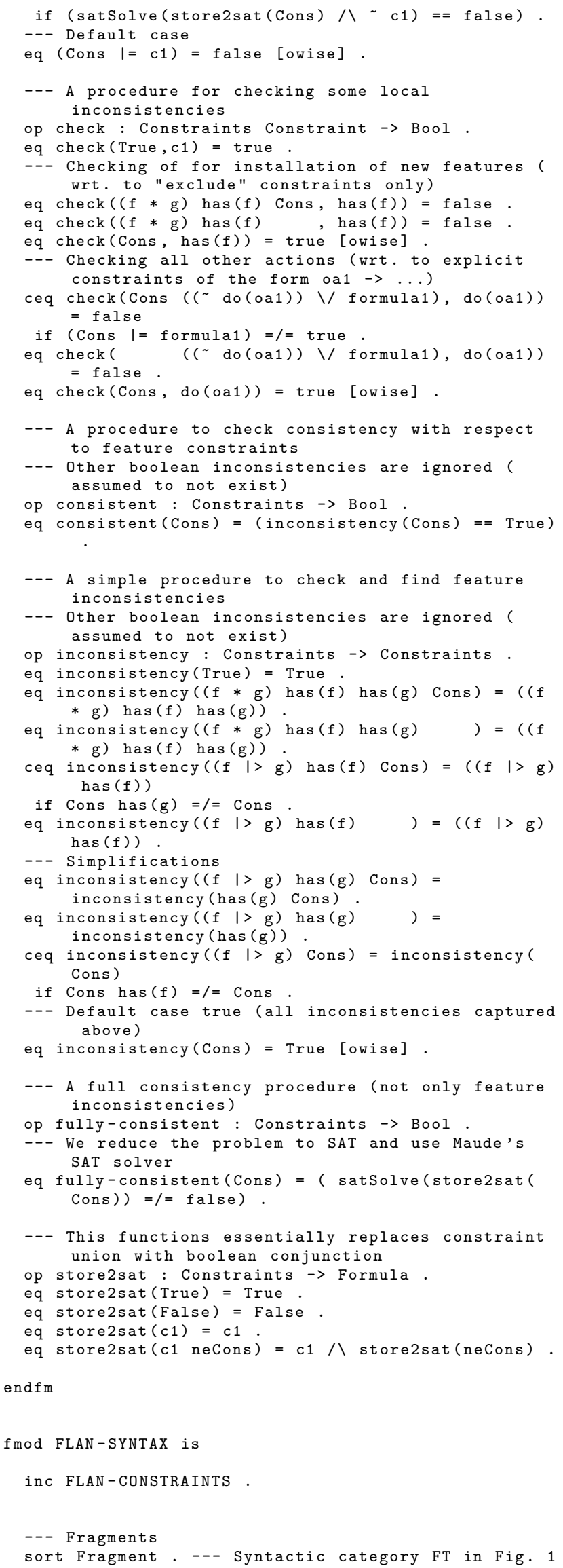

--- A full consistency procedure (not only feature inconsistencies) 


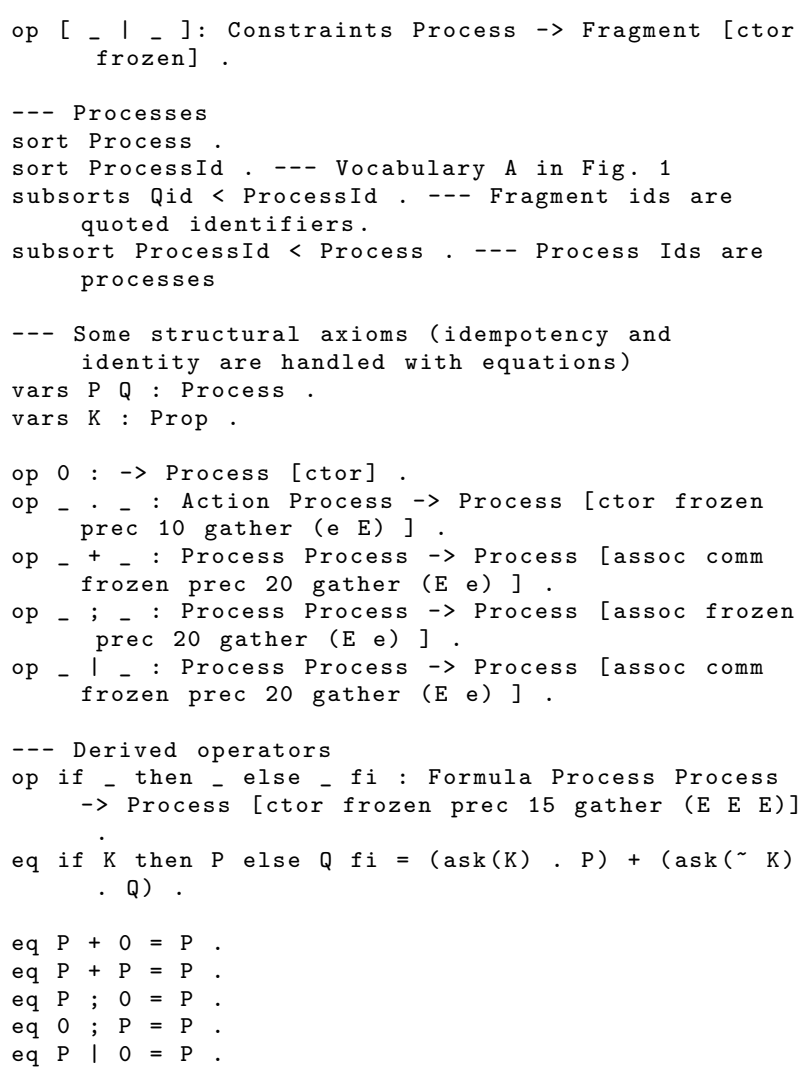

endfm

fmod FLAN-RECURSION is

inc FLAN-SYNTAX.

sorts ProcessDefinitions.

vars PId1 PId2 : ProcessId.

var P : Process.

vars PD1 PD2 : ProcessDefinitions.

--- We assume a global set of process definitions

endfm

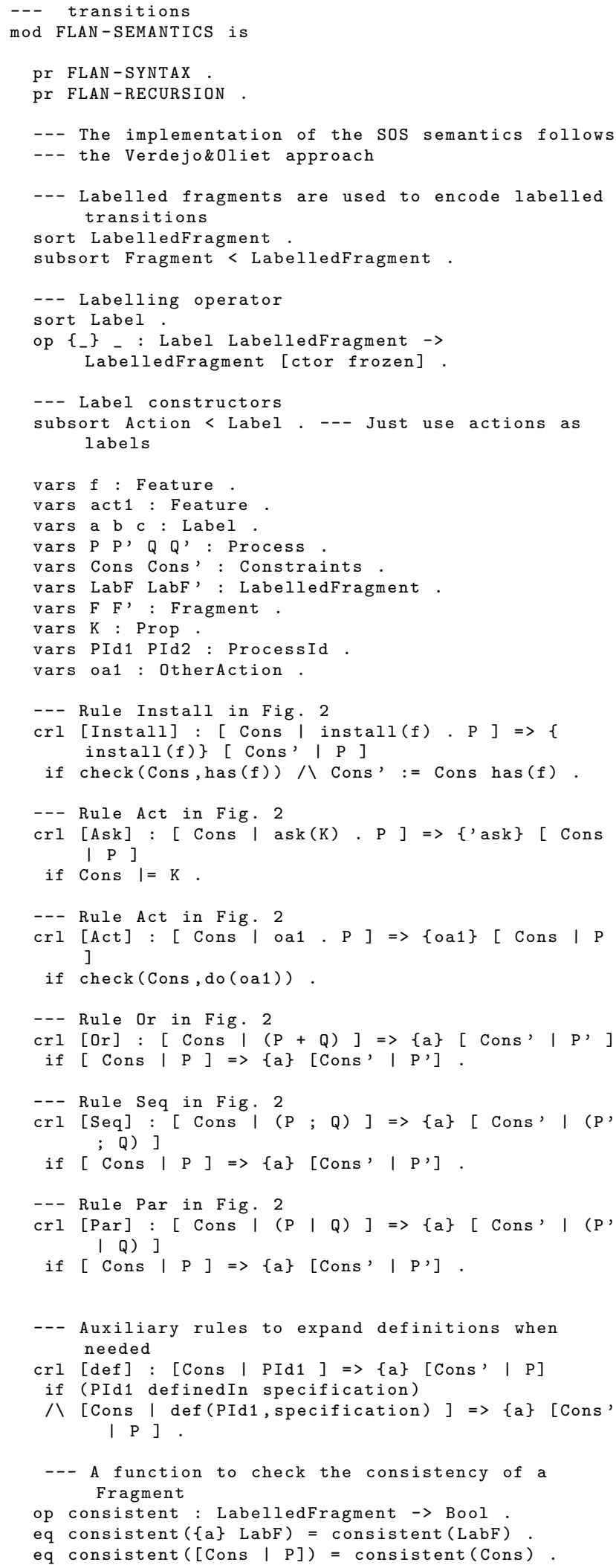




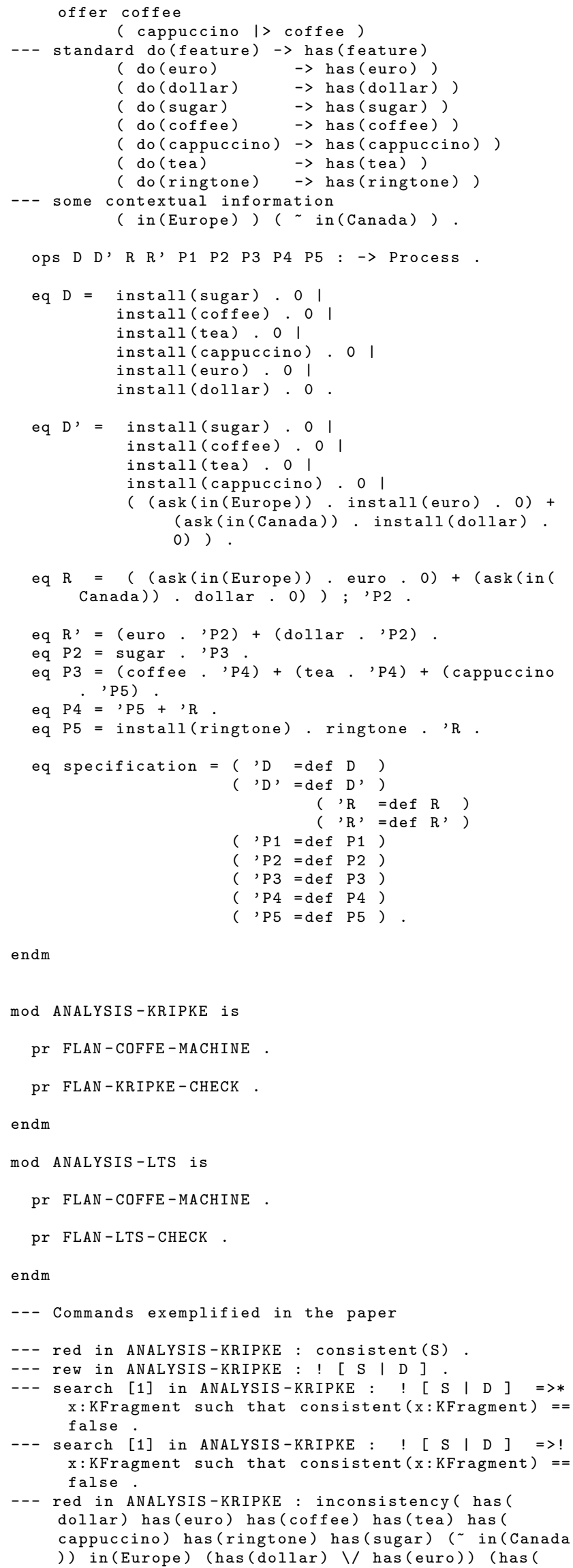

endm

$\bmod$ ANALYSIS-KRIPKE is

pr FLAN-COFFE-MACHINE.

pr FLAN-KRIPKE-CHECK.

endm

$\bmod$ ANALYSIS-LTS is

pr FLAN-COFFE-MACHINE.

pr FLAN-LTS-CHECK .

endm

--- Commands exemplified in the paper

--- red in ANALYSIS-KRIPKE : consistent(S).

--- rew in ANALYSIS-KRIPKE : $!$ [ S I D $]$

-- search [1] in ANALYSIS-KRIPKE : ! [ $\mathrm{S} \mid \mathrm{D}]=$ ] $\mathrm{x}$ : KFragment such that consistent $(\mathrm{x}: \mathrm{KFragment})==$ false

--- search [1] in ANALYSIS-KRIPKE : ! [ $S$ I $D$ ] $=>$ ! $\mathrm{x}$ : KFragment such that consistent $(\mathrm{x}:$ KFragment $)==$ false.

--- red in ANALYSIS-KRIPKE : inconsistency ( has ( dollar) has (euro) has (coffee) has (tea) has ( cappuccino) has (ringtone) has (sugar) ( in (Canada )) in(Europe) (has(dollar) \/ has(euro)) (has ( 